\title{
Is it possible to claim or refute sputum eosinophils $\geq 3 \%$ in asthmatics with sufficient accuracy using biomarkers?
}

\author{
Sophie F. Demarche ${ }^{1,2^{*}}$ (D) Florence N. Schleich ${ }^{1}$, Virginie A. Paulus ${ }^{1}$, Monique A. Henket ${ }^{1}$, Thierry J. Van Hees ${ }^{2}$
} and Renaud E. Louis ${ }^{1}$

\begin{abstract}
The concept of asthma inflammatory phenotypes has proved to be important in predicting response to inhaled corticosteroids. Induced sputum, which has been pivotal in the development of the concept of inflammatory phenotypes, is however not widely available. Several studies have proposed to use surrogate exhaled or blood biomarkers, like fractional exhaled nitric oxide (FENO), blood eosinophils and total serum immunoglobulin E (IgE). However, taken alone, each of these biomarkers has moderate accuracy to identify sputum eosinophilia. Here, we propose a new approach based on the likelihood ratio to study which thresholds of these biomarkers, taken alone or in combination, were able to rule in or rule out sputum eosinophils $\geq 3 \%$. We showed in a large population of 869 asthmatics that combining FENO, blood eosinophils and total serum IgE could accurately predict sputum eosinophils $\geq$ or $<3 \%$ in $58 \%$ of our population.
\end{abstract}

Keywords: Asthma, Phenotype, Biomarkers, Sputum, Blood, Eosinophils, Nitric oxide, Immunoglobulin E

\section{Introduction}

Asthma is a heterogeneous airway inflammatory disease. With the emergence of sputum analysis, the eosinophilic and non-eosinophilic phenotypes have been described, which were suggested to have different sensitivities to inhaled corticosteroids (ICS) [1]. It was indeed shown that eosinophilic asthmatics responded better to ICS in terms of symptoms, respiratory function and bronchial hyperresponsiveness than their non-eosinophilic counterparts [2,3].

Measuring sputum eosinophils in clinical practice is not applicable to the general population because it is technically demanding and time-consuming. To overcome this issue, user-friendly biomarkers like fractional exhaled nitric oxide (FENO), blood eosinophils and total serum immunoglobulin E (IgE) have been suggested as surrogate markers of airway eosinophilic inflammation [4]. Numerous thresholds for these biomarkers have been proposed to identify eosinophilic asthmatics [4-8], which may be confusing for the clinician. Moreover,

\footnotetext{
* Correspondence: Sophie.Demarche@ulg.ac.be

'Department of Respiratory Medicine, CHU Liege, GIGA I Research Group, University of Liege, Liege, Belgium

${ }^{2}$ Department of Clinical Pharmacy, Center for Interdisciplinary Research on Medicines, University of Liege, Liege, Belgium
}

each of these biomarkers taken alone has variable and moderate accuracy [4], that may be enhanced by combining them (like combining FENO and blood eosinophils [5]). Despite these efforts, there is currently no clear recommendation applicable to clinicians in their office practice to help them identify the eosinophilic status of their patients using the aforementioned biomarkers.

The purpose of our study was to assess whether several pragmatic thresholds of FENO, blood eosinophils and total IgE, taken alone or combined, were able to rule in or rule out sputum eosinophils $\geq 3 \%$. In order to answer this question, we have calculated the likelihood ratio (LR) for each corresponding threshold or combination of thresholds, on a large database of asthmatic patients. The LR is a measure of diagnostic accuracy combining both the sensitivity and specificity, which is particularly useful for the clinician, with values above 10 or below 0.1 allowing to rule in or rule out the "disease" (i.e. eosinophilic asthma in this study) with strong evidence [9].

\section{Methods}

We conducted a retrospective cross-sectional study on 869 asthmatics of varied severity recruited from the University Asthma Clinic of Liege, Belgium. Patients were eligible for 
the study if they had a visit with a successful measure of sputum eosinophils, FENO, blood eosinophils and total serum IgE (all performed on the same day) between January 2005 and September 2016. The study was approved by the ethics committee of the University Hospital of Liege (Ref 2016/276).

Asthma was diagnosed based on typical symptoms (wheezing, breathlessness, chest tightness, cough) and at least one of the following criteria: an improvement of $12 \%$ and $200 \mathrm{~mL}$ in forced expiratory volume in one second $\left(\mathrm{FEV}_{1}\right)$ following inhalation of $400 \mu \mathrm{g}$ salbutamol or a provocative concentration of methacholine causing a $20 \%$ fall in $\mathrm{FEV}_{1}(\mathrm{PC} 20 \mathrm{M})<16 \mathrm{mg} / \mathrm{mL}$. Sputum induction and processing were performed as previously described, using the whole expectorate technique [10]. The eosinophilic phenotype was defined as a sputum eosinophil count $\geq 3 \%$ [11]. FENO was measured at a flow rate of $50 \mathrm{~mL} / \mathrm{s}$ (NIOX, Aerocrine, Solna, Sweden). Blood samples of patients were analysed by the routine laboratory of the University Hospital of Liege.

Data were expressed as numbers and percentages for categorical variables and as median (first and third quartiles) or mean \pm standard deviation for continuous variables. Non-eosinophilic asthmatics were compared to eosinophilic asthmatics using a Pearson's chi-squared test for categorical variables, a Student's t-test for parametric continuous variables, and a Mann-Whitney test for non-parametric continuous variables. To assess the ability of FENO, blood eosinophils, total IgE or their combination to rule in or rule out eosinophilic asthma, we chose pragmatic thresholds for each biomarker based on previously published data [4-8], with 2 additional thresholds to improve the practicality of the tool: 150 blood eosinophils $/ \mu \mathrm{L}$ and $500 \mathrm{kU}$ total $\mathrm{IgE} / \mathrm{L}$. These thresholds are presented in Fig. 1. The LR calculation was performed in two parts. In the first part (presented in the right side of Fig. 1), we calculated the positive LRs to assess which threshold(s) was (were) able to rule in eosinophilic asthma. For each threshold or combination of thresholds, the positive LR was calculated as follows: \% of eosinophilic asthmatics whose value(s) of the biomarker(s) was (were) above the threshold(s)/\% of noneosinophilic asthmatics whose value(s) of the biomarker(s) was (were) above the threshold(s). This formula actually corresponds to: sensitivity/(1-specificity). Positive LR values above 10 allow to rule in eosinophilic asthma with strong evidence [9] and are highlighted by dark red in Fig. 1. In the second part (presented in the left side of Fig. 1), we calculated the negative LRs to assess which threshold(s) was (were) able to rule out eosinophilic asthma (i.e. to rule in non-eosinophilic asthma). For each threshold or combination of thresholds, the negative LR was calculated as follows: \% of eosinophilic asthmatics whose value(s) of the biomarker(s) was (were) below the threshold(s)/\% of non-eosinophilic asthmatics whose value(s) of the biomarker(s) was (were) below the threshold(s). This formula actually corresponds to: (1-sensitivity)/specificity. Negative LR values under 0.1 allow to rule out eosinophilic asthma (i.e. to rule in non-eosinophilic asthma) with strong evidence [9] and are highlighted by dark blue in Fig. 1. Positive LRs between 5 and 10 , and negative LRs between 0.1 and 0.2 give moderate evidence [8] to rule in or rule out eosinophilic asthma, and are only given in Fig. 1 for information. To ensure the reliability of the results, only subgroups including at least 20 patients were considered for the LR calculation. A $p$ value $<0.05$ was considered statistically significant. Statistical analysis was done using STATA version 13.0 (Statistical Software, College Station, TX: StataCorp LP).

\section{Results}

Our cohort included 869 patients. Patient characteristics of the total population and comparison between noneosinophilic and eosinophilic asthmatics are shown in Table 1. The prevalence of eosinophilic asthma was $43 \%$. Figure 1 represents the strength of the LR for each threshold of FENO, blood eosinophils and total IgE, taken alone or in combination. LRs above 10 give strong evidence to rule in eosinophilic asthma and are highlighted by dark red in Fig. 1 while LRs below 0.1 give strong evidence to rule out eosinophilic asthma (i.e. to rule in non-eosinophilic asthma) and are highlighted by dark blue in Fig. 1 [9]. The LRs for a single biomarker can be found in Fig. 1 where the values of the other two biomarkers are not taken into account. Likewise, the LRs for combinations of two biomarkers are found in the grid where the value of the third biomarker is not taken into account. For example, a patient with $<20 \mathrm{ppb}$ of FENO has not a strong prediction of non-eosinophilic asthma $(0.2<\mathrm{LR}<5)$, while a patient with $<20 \mathrm{ppb}$ of FENO and $<100$ blood eosinophils $/ \mu \mathrm{L}$ has a strong prediction of non-eosinophilic asthma $(\mathrm{LR}<0.1)$.

If we focus on clinically relevant LRs for the biomarkers taken alone, a value of FENO $>80$ ppb was associated with a LR above 10 (Fig. 1). In our population, $10 \%(89 / 869)$ of patients had a FENO $>80 \mathrm{ppb}$, of whom $79(89 \%)$ were actually eosinophilic. Likewise, a value of blood eosinophils $>1000$ cells $/ \mu \mathrm{L}$ was associated with a LR above 10 . In our study, $2 \%(20 / 869)$ of patients had $>1000$ blood eosinophils/ $\mu \mathrm{L}$, of whom 18 (90\%) were actually eosinophilic. This threshold was not shown in Fig. 1 because the subgroup it determined only included 20 patients and a combination with other biomarkers would necessarily lead to $<20$ patients per group. No threshold of FENO or blood eosinophils used as single biomarkers was associated with a LR below 0.1. As for total IgE, no threshold was able to 


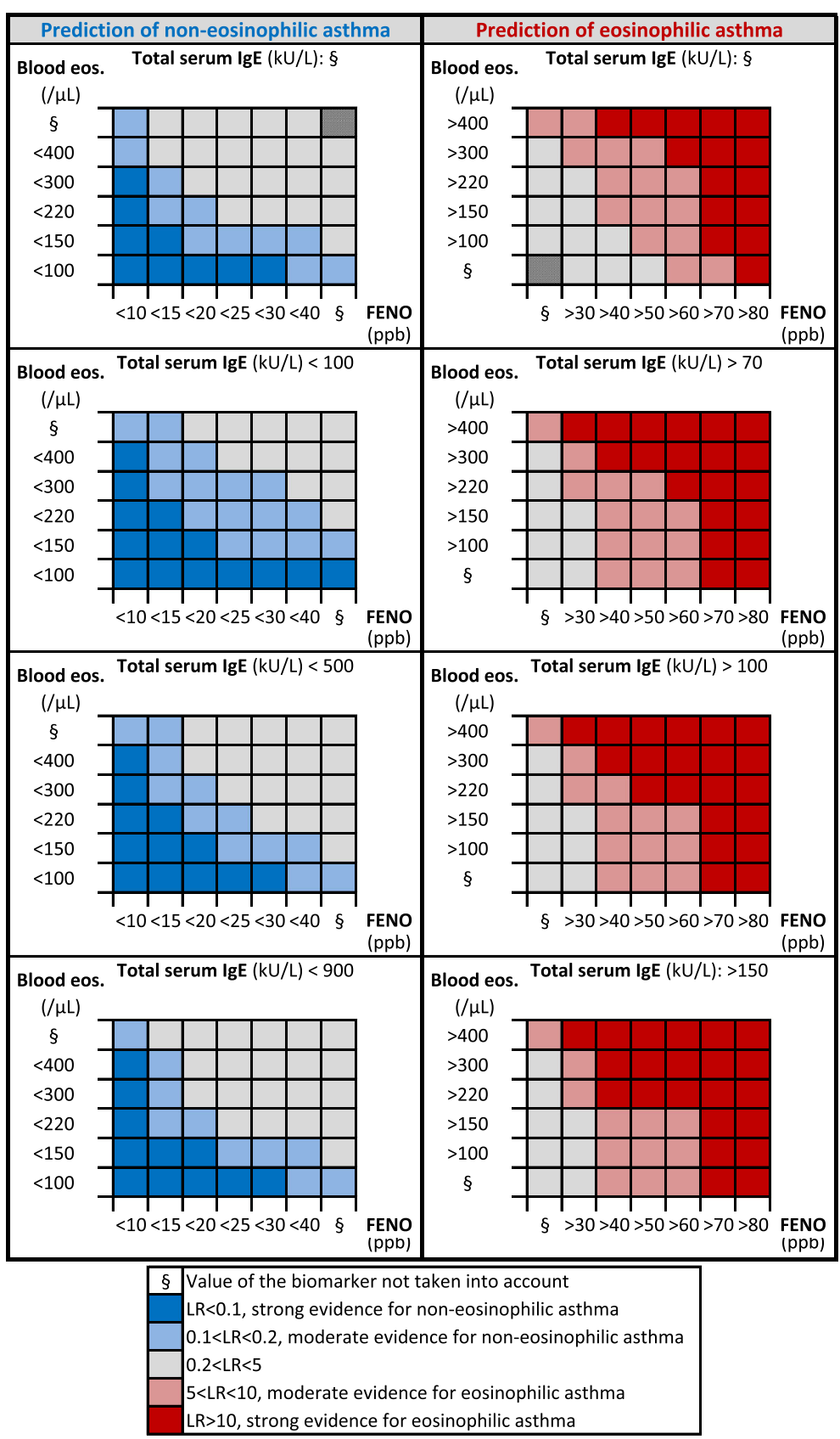

Fig. 1 Representation of the strength of the likelihood ratio to predict non-eosinophilic (left panel) or eosinophilic (right panel) asthma according to several thresholds of FENO, blood eosinophils, total IgE or their combination. Each square of the Figure represents a subgroup of at least 20 patients. Abbreviations: Blood eos blood eosinophils, FENO fractional exhaled nitric oxide, IgE immunoglobulin E

reach a LR above 10 or below 0.1 . When considering FENO, blood eosinophils or their combination without using total IgE (corresponding to the upper left panel and the upper right panel of Fig. 1), 45\% of the population (393/869) was included in at least one category associated with a LR above 10 or below 0.1 . Of these 393 patients, 352 (90\%) were properly classified into eosinophilic or noneosinophilic asthma. Finally, when considering FENO, blood eosinophils, total IgE or their combination (corresponding to the entire Fig. 1), 58\% of our population $(506 / 869)$ was included in at least one category associated with a LR above 10 or below 0.1 . Of these 506 patients, 440 (87\%) were properly classified into eosinophilic or non-eosinophilic asthma. The number of subjects with a strong evidence of eosinophilic or non-eosinophilic asthma was therefore significantly increased from 393 to 
Table 1 Patient characteristics

\begin{tabular}{|c|c|c|c|c|}
\hline & Total population & Non-eosinophilic asthmatics (NEA) & Eosinophilic asthmatics (EA) & $p$ (NEA vs EA) \\
\hline $\mathrm{N}$ & 869 & 495 & 374 & - \\
\hline Women, N (\%) & $505(58)$ & $314(63)$ & $191(51)$ & $<0.001$ \\
\hline Age, years & $49(35-61)$ & $46(33-59)$ & $51(38-63)$ & $<0.001$ \\
\hline $\mathrm{BMI}, \mathrm{kg} / \mathrm{m}^{2}$ & $26.2 \pm 5.2$ & $26.3 \pm 5.3$ & $26.2 \pm 5.0$ & 0.96 \\
\hline Atopy, N (\%) & $482(56)$ & $251(51)$ & $231(62)$ & 0.001 \\
\hline \multicolumn{5}{|l|}{ Smoking status, N (\%) } \\
\hline Non-smokers & $457(53)$ & $262(53)$ & $195(52)$ & \multirow[t]{3}{*}{0.049} \\
\hline Current smokers & $187(21)$ & $118(24)$ & $69(18)$ & \\
\hline Ex-smokers & $225(26)$ & $115(23)$ & $110(29)$ & \\
\hline $\mathrm{FEV}_{1}, \%$ predicted & $83.6 \pm 20.6$ & $86.6 \pm 19.8$ & $79.6 \pm 20.9$ & $<0.001$ \\
\hline $\mathrm{FEV}_{1} / \mathrm{FVC}, \%$ & $72.6 \pm 11.1$ & $74.4 \pm 10.6$ & $70.1 \pm 11.3$ & $<0.001$ \\
\hline $\mathrm{PC} 20 \mathrm{M}, \mathrm{mg} / \mathrm{mL}^{\mathrm{a}}$ & $2.9(0.7-13.0)$ & $3.7(0.9-15.0)$ & $1.8(0.5-9.8)$ & $<0.001$ \\
\hline Reversibility, \% & $7(2-12)$ & $6(2-11)$ & $8(3-14)$ & $<0.001$ \\
\hline ACQ score & $2.0 \pm 1.2$ & $1.9 \pm 1.1$ & $2.2 \pm 1.3$ & $<0.001$ \\
\hline AQLQ score & $4.5 \pm 1.4$ & $4.5 \pm 1.3$ & $4.5 \pm 1.4$ & 0.38 \\
\hline FENO, ppb & $23(13-45)$ & $17(12-29)$ & $41(21-72)$ & $<0.001$ \\
\hline Sputum eosinophils, \% of non-squamous cells & $1.8(0.2-10.8)$ & $0.2(0.0-1.0)$ & $13.2(6.5-35.0)$ & $<0.001$ \\
\hline Sputum neutrophils, \% of non-squamous cells & $53(28-76)$ & $64(39-84)$ & $41(22-59)$ & $<0.001$ \\
\hline Total serum IgE, kU/L & $121(37-328)$ & $79(23-227)$ & $200(80-472)$ & $<0.001$ \\
\hline Blood eosinophils, cells/uL & $188(109-328)$ & $137(80-217)$ & $290(189-507)$ & $<0.001$ \\
\hline Blood neutrophils, cells/uL & 4068 (3150-5402) & $4034(3058-5498)$ & $4078(3228-5312)$ & 0.58 \\
\hline \multicolumn{5}{|l|}{ ICS category, N (\%) } \\
\hline Steroid naive & $314(36)$ & $204(41)$ & $110(30)$ & \multirow[t]{4}{*}{0.003} \\
\hline Low dose $\mathrm{b}^{\mathrm{b}}$ & $126(15)$ & $71(14)$ & $55(15)$ & \\
\hline Medium dose $\mathrm{b}^{\mathrm{b}}$ & $182(21)$ & $97(20)$ & $85(23)$ & \\
\hline High dose ${ }^{b}$ & $242(28)$ & $121(25)$ & $121(33)$ & \\
\hline OCS therapy, N (\%) & $62(7)$ & $30(6)$ & $32(9)$ & 0.16 \\
\hline LABA, N (\%) & $530(61)$ & $279(56)$ & $251(67)$ & 0.001 \\
\hline LTRA, N (\%) & $220(25)$ & $130(26)$ & $90(24)$ & 0.46 \\
\hline Theophylline, N (\%) & $25(3)$ & $17(3)$ & $8(2)$ & 0.26 \\
\hline
\end{tabular}

Data available for 493 patients of the total population: 312 non-eosinophilic patients and 181 eosinophilic patients

${ }^{\mathrm{b}}$ Low dose ICS: $\leq 500 \mu \mathrm{g}$ per day; medium dose ICS: $>500-1000 \mu \mathrm{g}$ per day; high dose ICS: $>1000 \mu \mathrm{g}$ per day beclomethasone dipropionate - chlorofluorocarbon Abbreviations: $A C Q$ asthma control questionnaire, AQLQ asthma quality of life Questionnaire, BMI body mass index, FENO fractional exhaled nitric oxide, FEV 1 forced expiratory volume in $1 \mathrm{~s}$, FVC forced vital capacity, ICS inhaled corticosteroid, IgE immunoglobulin E, LABA long-acting $\beta 2$-agonist, LTRA leukotriene receptor antagonist, OCS oral corticosteroid, PC2OM provocative concentration of methacholine causing a $20 \%$ fall in $^{\mathrm{FEV}} \mathrm{F}_{1}$

506 patients when IgE levels were taken into account $(p<0.0001$ using the McNemar test), while keeping an accuracy of nearly $90 \%$.

In asthmatics untreated with ICS $(N=314), 189$ patients $(60 \%)$ were included in at least one category with a LR above 10 or below 0.1 in Fig. 1, of whom 169 (89\%) were correctly classified. In asthmatics treated with ICS $(N=555), 317$ patients $(57 \%)$ were included in at least one category with a LR above 10 or below 0.1 , of whom $271(85 \%)$ were correctly classified. The proportion of patients with a strong evidence of eosinophilic or noneosinophilic asthma and the correctness of the tool were not statistically different between patients untreated and treated with ICS ( $p=0.38$ and $p=0.20$, respectively).

\section{Discussion}

In our study, we showed that FENO and blood eosinophils used as single biomarkers were able to accurately predict sputum eosinophils $\geq 3 \%$ in a very small proportion of patients $(10 \%$ and $2 \%$, respectively), while IgE levels taken alone were unable to accurately predict the eosinophilic status. Overall, these results are consistent with previous studies reporting that these biomarkers are not sufficiently 
good predictors of the airway eosinophilic status when used as single surrogate markers $[4,12]$.

Hastie et al. showed that combining FENO and blood eosinophils did not accurately predict the eosinophilic phenotype in asthmatic patients, with an accuracy (defined as the percentage of subjects correctly classified by the test as having or not having eosinophilic asthma) of 55\% [12]. When using FENO and blood eosinophils, our data show that we can only achieve a satisfactory prediction in $45 \%$ of patients, which is not very different from what Hastie et al. reported. We also found that measuring total IgE may be of interest as the proportion of asthmatics accurately predicted rose from 45 to $58 \%$ of the population, which supports the idea that combining biomarkers is useful [13].

Our results highlight the fact that these three surrogate markers are not able to entirely replace the sputum analysis to determine the eosinophilic status of patients. However, we still believe that the use of these biomarkers is valuable in routine clinical practice. Their measure is easy to perform, well standardised, less costly than sputum analysis, and their results are rapidly available. Therefore, we may suggest measuring these biomarkers in asthmatic patients in whom the practitioner wants to know the eosinophilic phenotype. For the $58 \%$ of patients identified by the tool as having a strong evidence of eosinophilic or non-eosinophilic asthma, a sputum induction might be spared. However, for the remaining $42 \%$ of patients, a sputum induction would be recommended in a dedicated centre to identify their eosinophilic status.

One limitation of our study is its retrospective design. Moreover, the accuracy of the tool in Fig. 1 should also be studied in other centres performing sputum in asthmatic patients to assess its generalisability (external validity) [14]. Nevertheless, a strength of our study is that our asthmatic population encompassed the all spectrum of severity and our grid may therefore be applicable to a majority of patients seen in clinical practice. Although ICS are known to impact sputum and blood eosinophils as well as FENO [15], our data show that the tool is suitable for asthmatic patients regardless of treatment with ICS. Therefore, the Figure could be printed and kept in the pocket of the practitioner as a companion tool to profile the eosinophilic phenotype in asthma.

In conclusion, combining exhaled breath and blood biomarkers to identify airway eosinophilia appears to be a valuable attitude in clinical practice in around $60 \%$ of patients, leaving the remaining $40 \%$ with uncertain eosinophilic status, which should be investigated by a sputum analysis.

\section{Abbreviations}

FENO: fractional exhaled nitric oxide; $\mathrm{FEV}_{1}$ : forced expiratory volume in one second; ICS: inhaled corticosteroid; IgE: Immunoglobulin E; LR: likelihood ratio: PC20M: provocative concentration of methacholine causing a 20\% fall in $\mathrm{FEV}_{1}$

\section{Acknowledgements}

Not applicable.

Funding

With the support of a federal grant IAP (Interuniversity Attraction Poles Programme) P7/30.

\section{Availability of data and materials}

The datasets analysed during the current study are available from the corresponding author on reasonable request.

\section{Authors' contributions}

SD and RL designed the study; SD, FS, VP, MH and RL collected the data; $\mathrm{SD}$ analysed the data; SD, RL and TVH interpreted the data; SD and RL drafted the manuscript; FS, TVH, VP and MH revised the manuscript critically for important intellectual content; all authors read and approved the final manuscript.

\section{Ethics approval and consent to participate}

The study was approved by the ethics committee of the University Hospital of Liege (Ref 2016/276).

\section{Consent for publication}

Not applicable.

\section{Competing interests}

SD, FS, VP, MH: no competing interests. TVH: grants from Amgen, outside the submitted work. RL: grants and personal fees from GSK, Chiesi, Astra Zeneca, Novartis, outside the submitted work.

\section{Publisher's Note}

Springer Nature remains neutral with regard to jurisdictional claims in published maps and institutional affiliations.

Received: 4 May 2017 Accepted: 25 June 2017

Published online: 03 July 2017

\section{References}

1. Brightling CE. Clinical applications of induced sputum. Chest. 2006;129: 1344-8.

2. Bacci E, Cianchetti S, Bartoli M, Dente FL, Di Franco A, Vagaggini B, et al. Low sputum eosinophils predict the lack of response to beclomethasone in symptomatic asthmatic patients. Chest. 2006:129:565-72.

3. Pavord ID, Brightling CE, Woltmann G, Wardlaw AJ. Non-eosinophilic cor ticosteroid unresponsive asthma. Lancet. 1999:353:2213-4.

4. Korevaar DA, Westerhof GA, Wang J, Cohen JF, Spijker R, Sterk PJ, et al. Diagnostic accuracy of minimally invasive markers for detection of airway eosinophilia in asthma: a systematic review and meta-analysis. Lancet Respir Med. 2015;3:290-300.

5. Westerhof GA, Korevaar DA, Amelink M, de Nijs SB, de Groot JC, Wang J, et al. Biomarkers to identify sputum eosinophilia in different adult asthma phenotypes. Eur Respir J. 2015;46:688-96.

6. Schleich FN, Seidel L, Sele J, Manise M, Quaedvlieg V, Michils A, et al. Exhaled nitric oxide thresholds associated with a sputum eosinophil count $\geq 3 \%$ in a cohort of unselected patients with asthma. Thorax. 2010;65:1039-44.

7. Wagener AH, de Nijs SB, Lutter R, Sousa AR, Weersink EJM, Bel EH, et al. External validation of blood eosinophils, $F E(N O)$ and serum periostin as surrogates for sputum eosinophils in asthma. Thorax. 2015;70:115-20.

8. Taylor DR. Using biomarkers in the assessment of airways disease. J Allergy Clin Immunol. 2011;128:927-34. quiz 935-936

9. Deeks JJ, Altman DG. Diagnostic tests 4: likelihood ratios. BMJ. 2004;329: 168-9.

10. Demarche SF, Schleich FN, Paulus VA, Henket MA, Van Hees TJ, Louis RE. Asthma Control and Sputum Eosinophils: A Longitudinal Study in Daily Practice. J Allergy Clin Immunol Pract. 2017. http://dx.doi.org/10.1016/j.jaip. 2017.01.026.

11. Louis R, Godinas L, Schleich F. Induced sputum - towards normal values. In: Loukides S, Kostikas K, Barnes PJ, editors. Non invasive assessment of airways inflammation in asthma and COPD. Athens: Paschalidis Medical Publications; 2011. p. 113-23. 
12. Hastie AT, Moore WC, Li H, Rector BM, Ortega VE, Pascual RM, et al. Biomarker surrogates do not accurately predict sputum eosinophil and neutrophil percentages in asthmatic subjects. J Allergy Clin Immunol. 2013;132:72-80.e12.

13. Coumou H, Bel EH. Improving the diagnosis of eosinophilic asthma. Expert Rev Respir Med. 2016;10:1093-103.

14. Justice AC, Covinsky KE, Berlin JA. Assessing the generalizability of prognostic information. Ann Intern Med. 1999;130:515-24.

15. Szefler SJ, Wenzel S, Brown R, Erzurum SC, Fahy JV, Hamilton RG, et al. Asthma outcomes: biomarkers. J Allergy Clin Immunol. 2012;129:S9-23.

Submit your next manuscript to BioMed Central and we will help you at every step:

- We accept pre-submission inquiries

- Our selector tool helps you to find the most relevant journal

- We provide round the clock customer support

- Convenient online submission

- Thorough peer review

- Inclusion in PubMed and all major indexing services

- Maximum visibility for your research

Submit your manuscript at www.biomedcentral.com/submit 